\title{
THE SMALLEST PROPER CONGRUENCE ON $S(X)$
}

\author{
by K. H. HOFMANN AND K. D. MAGILL, JR.
}

(Received 11 May, 1987; revised 29 September, 1987)

1. Introduction. $S(X)$ is the semigroup of all continuous selfmaps of the topological space $X$ and for any semigroup $S$, $\operatorname{Cong}(S)$ will denote the complete lattice of congruences on $S$. Cong $(S)$ has a zero $Z$ and a unit $U$. Specifically, $Z=\{(a, a): a \in S\}$ and $U=S \times S$. Evidently, $Z$ and $U$ are distinct if $S$ has at least two elements. By a proper congruence on $S$ we mean any congruence which differs from each of these. Since $S(X)$ has more than one element when $X$ is nondegenerate, we will assume without further mention that the spaces we discuss in this paper have more than one point. We observed in [4] that there are a number of topological spaces $X$ such that $S(X)$ has a largest proper congruence, that is, Cong $(S(X))$ has a unique dual atom which is greater than every other proper congruence on $S(X)$. On the other hand, we also found out in [5] that it is also common for $S(X)$ to fail to have a largest proper congruence. We will see that the situation is quite different at the other end of the spectrum in that it is rather rare for $S(X)$ not to have a smallest proper congruence. In other words, for most spaces $X$, Cong $(S(X))$ has a unique atom which is smaller than every other proper congruence.

In Section 2, we study a rather general class of transformation semigroups which includes all $S(X)$. We show that the problem of getting information about the atoms in the complete lattice of congruences on one of these semigroups can be reduced to examining a certain lattice of equivalence relations on $X$. It turns out that there is a natural correspondence between the atoms of the two lattices and we use this fact to get a necessary and sufficient condition for any semigroup within this class to have a smallest proper congruence. The results of Section 2 are then applied in Section 3 to the semigroups of topological spaces and we are able to show, for example, that if $X$ is either totally separated or is completely regular, Hausdorff and contains an arc then $S(X)$ has a smallest proper congruence. We show further that the semigroup of every finite space has a smallest proper congruence. All this indicates that semigroups of continuous selfmaps which do not have smallest proper congruences are the exception rather than the rule. However, there are examples and they are provided by what have come to be the standard counter-examples in this business, namely, de Groot's spaces. The nature of the smallest proper congruence is related to the connectedness of $X$ and this is explored in Section 4 . If a semigroup $S$ has a nontrivial kernel $K$ (i.e., smallest two-sided ideal) then the Rees factor congruence $\pi(K)$, obtained by identifying all elements in $K$ to a point, is a congruence on $S$. Now $\pi(K)$ can turn out to be the smallest proper congruence on $S$. We show that if $X$ is completely regular and Hausdorff and contains an arc, then $\pi(K(X))$ is the smallest proper congruence on $S(X)$ if and only if $X$ is pathwise connected where $K(X)$ is the kernel of $S(X)$. We then produce another class of spaces with the property that for any such space $X$, the two conditions just mentioned are also equivalent to the

Glasgow Math. J. 30 (1988) 301-313. 
condition that $S(X)$ have no prime ideals and these, in turn, are all equivalent to the condition that $S(X)$ have a largest proper congruence.

2. Some general results. Throughout the remainder of the paper, the symbol $\langle x\rangle$ will be used to denote the constant function which maps all of $X$ into the point $x$. Let $X$ be a set and let $T(X)$ be any subsemigroup of the semigroup of all functions mapping $X$ into $X$ which contains the identity map and all constant maps.

Definition (2.1). An equivalence relation $\rho$ on $X$ will be called a $T$-equivalence if $(f(x), f(y)) \in \rho$ for all $(x, y) \in \rho$ and $f \in T(X)$. The collection of all $T$-equivalences on $X$ will be denoted by $T$ eq $(X)$.

Definition (2.2). For each $R \in \operatorname{Cong}(T(X))$, we let

$$
\gamma(R)=\{(x, y) \in X \times X:(\langle x\rangle,\langle y\rangle) \in R\} .
$$

The next result is an immediate consequence of the fact that $\langle f(x)\rangle=f \circ\langle x\rangle$.

Lemma (2.3). $\gamma(R)$ is a T-equivalence on $X$ for each $R \in \operatorname{Cong}(T(X))$.

Since arbitrary intersections of $T$-equivalences are $T$-equivalences, we also have

LEMMA (2.4). Teq $(X)$ is a complete lattice.

For any set $Y$ we will denote the diagonal of $Y \times Y$ by $\Delta(Y)$. That is, $\Delta(Y)=$ $\{(y, y): y \in Y\}$.

Definition (2.5). For each $\rho \in T \mathrm{eq}(X)$, we let

$$
C(\rho)=\{(\langle x\rangle,\langle y\rangle):(x, y) \in \rho\} \cup \Delta(T(X)) .
$$

Lemma (2.6). $C(\rho)$ is a congruence on $T(X)$ for each $\rho \in T$ eq $(X)$.

Proof. It is evident that $C(\rho)$ is an equivalence relation. Moreover, composition is compatible on the right because constant functions are left zeros and compatible on the left since $\rho$ is a $T$-equivalence.

Now we consider the two functions $C$ and $\gamma$ which map $T$ eq $(X)$ into $\operatorname{Cong}(T(X))$ and $\operatorname{Cong}(T(X))$ into $T \mathrm{eq}(X)$ respectively. The next observation is an immediate consequence of the definitions. $T \operatorname{eq}(X)$.

LEMma (2.7). $C$ and $\gamma$ are both monotone functions and $\gamma \circ C$ is the identity map on

Lemma (2.8). Let $\rho \in T \mathrm{eq}(X)$ and $R \in \operatorname{Cong}(T(X))$. Then $\rho \subseteq \gamma(R)$ if and only if $C(\rho) \subseteq R$.

Proof. Suppose $\rho \subseteq \gamma(R)$ and take $(\langle x\rangle,\langle y\rangle) \in C(\rho)$. Then $(x, y) \in \rho$. Thus, $(x, y) \in \gamma(R)$ which means $(\langle x\rangle,\langle y\rangle) \in R$. On the other hand, suppose $C(\rho) \subseteq R$ and take any $(x, y) \in \rho$. Then $(\langle x\rangle,\langle y\rangle) \in C(\rho)$ and hence $(\langle x\rangle,\langle y\rangle) \in R$. Evidently, $(x, y) \in$ $\gamma(R)$. 
(By this lemma, $(\gamma, C)$ is a covariant Galois connection between Cong $(T(X))$ and $T$ eq $(X)$.)

For each pair of distinct points $x, y \in X$, define

$$
\eta(x, y)=\{(f(x), f(y)): f \in T(X)\} \cup\{(f(y), f(x)): f \in T(X)\}
$$

and let $\eta_{e}(x, y)$ be the smallest equivalence relation on $X$ containing $\eta(x, y)$.

Lemma (2.9). $\eta_{e}(x, y)$ is a T-equivalence on $X$.

Proof. Let $(a, b) \in \eta_{e}(x, y)$. Since $\eta_{e}(x, y)$ is the transitive closure of $\eta(x, y)$, there exists a sequence of points $\left\{c_{j}\right\}_{j=1}^{N}$ such that $\left(a, c_{1}\right) \in \eta(x, y),\left(c_{N}, b\right) \in \eta(x, y)$ and $\left(c_{j}, c_{j+1}\right) \in \eta(x, y)$ for $1 \leq j \leq N-1$. Then there exist $N+1$ functions $\left\{f_{j}\right\}_{j=1}^{N+1}$ in $T(X)$ such that $f_{1}$ maps $\{x, y\}$ onto $\left\{a, c_{1}\right\}, f_{N+1}$ maps $\{x, y\}$ onto $\left\{c_{N}, b\right\}$ and $f_{j}$ maps $\{x, y\}$ onto $\left\{c_{j-1}, c_{j}\right\}$ for $2 \leq j \leq N$. Then for any $g \in T(X), g \circ f_{1}$ maps $\{x, y\}$ onto $\left\{g(a), g\left(c_{1}\right)\right\}$, $g \circ f_{N+1}$ maps $\{x, y\}$ onto $\left\{g\left(c_{N}\right), g(b)\right\}$ and $g \circ f_{j}$ maps $\{x, y\}$ onto $\left\{g\left(c_{j-1}\right), g\left(c_{j}\right)\right\}$ for $2 \leq j \leq N$. It follows that $(g(a), g(b)) \in \eta_{e}(x, y)$ which means $\eta_{e}(x, y)$ is a $T$-equivalence.

Lemma (2.10). Let $A$ be an atom of $\operatorname{Cong}(T(X))$, let $(f, g) \in A$ and suppose $f \neq g$. Then both $f$ and $g$ are constant functions.

Proof. Since $f \neq g$, there exists an $a \in X$ such that $f(a) \neq g(a)$. Let $x=f(a)$ and $y=g(a)$. Then $\eta_{e}(x, y) \neq \Delta(X)$ and so $C\left(\eta_{e}(x, y)\right) \neq \Delta(T(X))$. But it readily follows that $C\left(\eta_{e}(x, y)\right) \subseteq A$ and since $A$ is an atom, we must have $C\left(\eta_{e}(x, y)\right)=A$. It is now evident from Definition (2.5) that $f$ and $g$ must be constant functions.

We observed in Lemma (2.7) that $\gamma^{\circ} C$ is the identity map on $T e q(X)$. It follows immediately from this that $C$ must be injective on $T$ eq $(X)$ and $\gamma$ must be surjective from $\operatorname{Cong}(T(X))$ onto $T \mathrm{eq}(X)$. As one would expect, however, $C$ is far from being surjective and $\gamma$ is far from being injective. But if we restrict these maps to the atoms of the two lattices, the situation is quite different. Denote by $A \operatorname{Cong}(T(X))$ the collection of atoms of Cong $(T(X))$ and, similarly, denote by $A T \mathrm{eq}(X)$ the collection of atoms of $T \mathrm{eq}(X)$.

THEOREM (2.11). $\gamma$ maps $A \operatorname{Cong}(T(X))$ into $A T \mathrm{eq}(X)$ and $C$ maps $A T \mathrm{eq}(X)$ into $A \operatorname{Cong}(T(X))$. Moreover, $\gamma \circ C$ is the identity map on $A T \operatorname{eq}(X)$ and $C \circ \gamma$ is the identity map on $A T \mathrm{eq}(X)$ so that $\gamma$ actually maps $A \operatorname{Cong}(T(X))$ bijectively onto $\dot{A} T \mathrm{eq}(X)$ and, similarly, $C$ maps $A T \mathrm{eq}(X)$ bijectively onto $A \operatorname{Cong}(T(X))$.

Proof. Let $A \in A \operatorname{Cong}(T(X))$ and suppose $\rho \subseteq \gamma(A)$ where $\rho \in T$ eq $(X)$. Then $C(\rho) \subseteq A$ by Lemma (2.8) and we have either $C(\rho)=\Delta(T(X))$ or $C(\rho)=A$. In the former case, $\rho=\Delta(X)$ while in the latter, $\rho=\gamma(C(\rho))=\gamma(A)$ by Lemma (2.7). Thus, $\gamma(A)$ is an atom.

Now let $\alpha \in A T \operatorname{eq}(X)$ and suppose that $R \subseteq C(\alpha)$ where $R \in \operatorname{Cong}(T(X))$. Then $\gamma(R) \subseteq \alpha$ in view of Lemma (2.7) which means that either $\gamma(R)=\Delta(X)$ or $\gamma(R)=\alpha$. In the former case, it follows easily that $R=\Delta(T(X))$. On the other hand, if $\gamma(R)=\alpha$, it follows from Lemma (2.8) that $C(\alpha) \subseteq R$ and thus $C(\alpha)=R$. Hence, $C(\alpha)$ is an atom. 
We have already observed that $\gamma \circ C$ is the identity on all of $T$ eq $(X)$ so it is certainly the identity on $A T$ eq $(X)$. It only remains for us to show that $C \circ \gamma$ is the identity on $A$ Cong $(T(X))$. We first verify that

$$
\gamma \text { is injective on } A \text { Cong }(T(X)) \text {. }
$$

Let $A, B \in A \operatorname{Cong}(T(X))$ and suppose $\gamma(A)=\gamma(B)$. Let $(f, g) \in A$ and we may assume that $f \neq g$. Then $f=\langle x\rangle$ and $g=\langle y\rangle$ for distinct points $x, y \in X$ by Lemma (2.10). Thus, $(x, y) \in \gamma(A)$ which means $(x, y) \in \gamma(B)$. But this implies $(f, g)=(\langle x\rangle,\langle y\rangle) \in B$ and we have shown that $A \subseteq B$. Since $B$ is an atom, $A=B$ and (2.11.1) has been verified. Now we know that for any $A \in A \operatorname{Cong}(T(X)), C \circ \gamma(A) \in A \operatorname{Cong}(T(X))$. Let $C \circ \gamma(A)=B$. Since $\gamma \circ C$ is the identity on $A T \operatorname{eq}(X)$, it follows that $\gamma(A)=\gamma(B)$ and hence $A=B$ by (2.11.1).

Lemma (2.12). Let $R \in \operatorname{Cong}(T(X))$. Then $R=\Delta(T(X))$ if and only if $\gamma(R)=\Delta(X)$.

Proof. It is immediate that if $\gamma(R) \neq \Delta(X)$, then $R \neq \Delta(T(X))$. Suppose $R \neq$ $\Delta(T(X))$. Then $(f, g) \in R$ for two distinct functions $f$ and $g$ and we have $f(x) \neq g(x)$ for some $x \in X$. Then

$$
(\langle f(x)\rangle,\langle g(x)\rangle)=(f \circ\langle x\rangle, g \circ\langle x\rangle) \in R,
$$

which means $(f(x), g(x)) \in \gamma(R)$. Thus, $\gamma(R) \neq \Delta(X)$.

Definition (2.13). We will say that a lattice is weakly atomic if every nonzero element has an atom below it.

Theorem (2.14). Cong $(T(X))$ is weakly atomic if and only if $T \operatorname{eq}(X)$ is weakly atomic.

Proof. Suppose $T \operatorname{eq}(X)$ is weakly atomic and let $R \in \operatorname{Cong}(T(X))$, where $R \neq$ $\Delta(T(X))$. By Lemma (2.12) we have $\gamma(R) \neq \Delta(X)$ and so there is an atom $\alpha \in T$ eq $(X)$ such that $\alpha \subseteq \gamma(R)$. Then $C(\alpha) \subseteq R$ by Lemma (2.8) and $C(\alpha)$ is an atom by Theorem (2.11). Now suppose $\operatorname{Cong}(T(X))$ is weakly atomic and let $\rho \in T$ eq $(X)$ where $\rho \neq \Delta(X)$. Then $C(\rho) \neq C(\Delta(X))=\Delta(T(X))$ since $C$ is injective. Thus, $A \subseteq C(\rho)$ for some atom $A \in \operatorname{Cong}(T(X))$ and it follows that $\gamma(A) \subseteq \gamma(C(\rho))=\rho$ from Lemma (2.7). Moreover, it follows from Theorem (2.11) that $\gamma(A)$ is an atom of $T$ eq $(X)$.

If a weakly atomic lattice has exactly one atom, then it must lie below every other nonzero element. Our next result is therefore an immediate consequence of Theorems (2.11) and (2.14).

COROLlaRY (2.15). $T(X)$ has a smallest proper congruence if and only if $X$ has a smallest proper $T$-equivalence. Furthermore, if $A$ is the smallest proper congruence on $T(X)$ then $\gamma(A)$ is the smallest proper $T$-equivalence on $X$. Similarly, if $\alpha$ is the smallest proper $T$-equivalence on $X$, then $C(\alpha)$ is the smallest proper congruence on $T(X)$.

As one might expect, if $T \operatorname{eq}(X)$ does have an atom, then it must be of the form $\eta_{e}(x, y)$. As one might also expect, not all $T$-equivalence of that form are atoms. In our next result, we characterize the atoms of $T$ eq $(X)$. 
Theorem (2.16). $A \in T e q(X)$ is an atom if and only if it satisfies the following two conditions.

$$
A=\eta_{e}(x, y) \text { for distinct } x, y \in X \text {. }
$$

If $(a, b) \in \eta_{e}(x, y)$ and $a \neq b$, then there exists a sequence of points $\left\{z_{j}\right\}_{j=1}^{N}$ in

$X$ and a sequence of functions $\left\{f_{j}\right\}_{j=1}^{N+1}$ from $T(X)$ such that $f_{1}\{a, b\}=\left\{x, z_{1}\right\}$, $f_{N+1}\{a, b\}=\left\{z_{N}, y\right\}$ and $f_{j}\{a, b\}=\left\{z_{j-1}, z_{j}\right\}$ for $2 \leq j \leq N$.

Proof. Suppose (2.16.1) and (2.16.2) are satisfied and let $B \subseteq \eta_{e}(x, y)$ where $B \neq \Delta(X)$. Then $(a, b) \in B$ where $a \neq b$ and it follows that $\eta_{e}(a, b) \subseteq B \subseteq \eta_{e}(x, y)$. Moreover, since $(a, b) \in \eta_{e}(x, y)$, it follows from (2.16.2) that $(x, y) \in \eta_{e}(a, b)$ and this implies $\eta_{e}(x, y) \subseteq \eta_{e}(a, b)$. Consequently, $B=\eta_{e}(x, y)$ and we see that $\eta_{e}(x, y)$ is an atom.

Suppose, conversely, that $A$ is an atom of $T \operatorname{eq}(X)$. Then $(x, y) \in A$ where $x \neq y$. Since $\eta_{e}(x, y) \subseteq A$, we must in fact, have $\eta_{e}(x, y)=A$. Now let $(a, b) \in \eta_{e}(x, y)$ where $a \neq b$. Then $\eta_{e}(a, b) \subseteq \eta_{e}(x, y)$ and again we must have $\eta_{e}(a, b)=\eta_{e}(x, y)$. Then $(x, y) \in \eta_{e}(a, b)$ and since $\eta_{e}(a, b)$ is the transitive closure of $\eta(a, b)$, condition (2.16.2) is satisfied.

Example (2.17.1). Let $R$ denote the space of real numbers and let $T(R)$ be the semigroup consisting of all constant functions together with all increasing homeomorphisms $h$ from $R$ onto $R$ which fix 0 . In this case $T$ eq $(R)$ has exactly two atoms and they are $\eta_{e}(1,2)$ and $\eta_{e}(-1,-2)$. Note that

$$
\eta(1,2)=\{(x, y): x>0 \text { and } y>0\}
$$

so that $\eta_{e}(1,2)=\eta(1,2) \cup \Delta(R)$. It is immediate that $(2.16 .2)$ is satisfied so that $\eta_{e}(1,2)$ is an atom. It follows in the same manner that $\eta_{e}(-1,-2)$ is an atom as well. To see that there are no other atoms, consider $\eta_{e}(x, y)$ which is distinct from both $\eta_{e}(1,2)$ and $\eta_{e}(-1,-2)$. There are essentially two possibilities: (1) $x=0$ and $y \neq 0$ and (2) $x<0<y$. Suppose (1) holds and $y>0$. Then $(1,2) \in \eta_{e}(x, y)$ but no sequences of points and functions satisfying (2.16.2) exist since all nonconstant functions of $T(R)$ fix 0 . The same is true if $y<0$. If (2) holds, then $(1,2) \in \eta_{e}(x, y)$ but $(2.16 .2)$ cannot be satisfied in this case either.

ExAmple (2.17.2). Let $R$ denote the space of positive real numbers, let $f(x)=x^{3}$ and this time let $T(R)$ denote the subsemigroup of $S(R)$ which is generated by $f$ together with the identity map and all the constant functions. In this example, Teq $(R)$ contains no atoms whatsoever. Consider any $\eta_{e}(x, y)$. We may suppose that $x<y$. Then $\left(x^{3}, y^{3}\right) \in$ $\eta_{e}(x, y)$ but there exist no sequences of points and functions satisfying (2.16.2).

In view of Corollary (2.15) the problem of determining whether or not $T(X)$ has a smallest proper congruence and when it does, determining just what that congruence is, has been reduced to the problem of determining when $X$ has a smallest proper $T$-equivalence and then determining the nature of that equivalence. We now have everything we need to solve the latter problem. For a given semigroup $T(X)$, let

$$
\sigma=\bigcap\left\{\eta_{e}(x, y): x, y \in X \text { and } x \neq y\right\} \text {. }
$$


Evidently, $\sigma$ depends upon the semigroup $T(X)$ and so might be more properly denoted by $\sigma(T(X))$. Nevertheless, it will always be apparent just what semigroup is under consideration so we will use the simpler notation $\sigma$. Moreover the symbol $\sigma$ will be used exclusively to denote the $T$-equivalence we have just defined.

THEOREM (2.18). $X$ has a smallest proper T-equivalence if and only if $\sigma \neq \Delta(X)$. Moreover, when $X$ does have a smallest proper $T$-equivalence, that equivalence is $\sigma$.

Proof. Suppose $X$ has a smallest proper $T$-equivalence $\alpha$. Then according to Theorem (2.16), $\alpha=\eta_{e}(a, b)$ for distinct points $a$ and $b$ and we have $\sigma \subseteq \alpha$. On the other hand, $\alpha \subseteq \eta_{e}(x, y)$ for all distinct $x$ and $y$ so that we also have $\alpha \subseteq \sigma$. Thus, $\sigma=\alpha \neq \Delta(X)$.

Suppose, conversely that $\sigma \neq \Delta(X)$ and suppose also that $\rho \neq \Delta(X)$. Then $(a, b) \in \rho$ where $a \neq b$ and so $\eta_{e}(a, b) \subseteq \rho$. Thus, $\sigma \subseteq \rho$ and $\sigma$ is the smallest proper $T$-equivalence on $X$.

We next introduce another equivalence relation on $X$ which, for many semigroups of the form $T(X)$, coincides with the $T$-equivalence $\sigma$. Let

$$
\begin{aligned}
\mu= & \{(x, y) \in X \times X \text { : for each pair of distinct points } a, b \in X, \\
& \text { some } f \in T(X) \text { maps }\{a, b\} \text { onto }\{x, y\}\}
\end{aligned}
$$

and let $\mu_{e}$ denote the smallest equivalence relation on $X$ containing $\mu$.

TheOREM (2.19). Either $\mu_{e}=\Delta(X)$ or $\mu_{e}=\sigma$.

Proof. It is sufficient to show that $\mu_{e} \subseteq \eta_{e}(x, y)$ for distinct $x$ and $y$. Let $(a, b) \in \mu_{e}$. Since $\mu_{e}$ is the transitive closure of $\mu$, there exists a sequence of points $\left\{c_{j}\right\}_{j=1}^{N}$ and a sequence of functions $\left\{f_{j}\right\}_{j=1}^{N+1}$ from $T(X)$ such that $f_{1}\{x, y\}=\left\{a, c_{i}\right\}, f_{N+1}\{x, y\}=\left\{c_{N}, b\right\}$ and $f_{j}\{x, y\}=\left\{c_{j-1}, c_{j}\right\}$ for $2 \leq j \leq N$. Thus, both $\left(a, c_{1}\right)$ and $\left(c_{N}, b\right)$ belong to $\eta(x, y)$ and the same is true of $\left(c_{j-1}, c_{j}\right)$ for $2 \leq j \leq N$. It follows that $(a, b) \in \eta(x, y)$.

CoRollary (2.20). Suppose there exists a pair of distinct points $a, b \in X$ such that for each pair of distinct points $x, y \in X$, some $f \in T(X)$ maps $\{x, y\}$ onto $\{a, b\}$. Then $X$ has $a$ smallest proper $T$-equivalence.

Proof. $\mu_{e} \neq \Delta(X)$ since $(a, b) \in \mu_{e}$.

In order that $T(X)$ have a smallest proper congruence, it is necessary and sufficient that $\sigma \neq \Delta(X)$. The relation $\mu_{e}$ seems to be more convenient to deal with than the relation $\sigma$ and, in fact, most of the time we show that a semigroup has a smallest proper congruence, we are able to do it by showing that $\mu_{e} \neq \Delta(X)$. In other words, in most of our applications, $\mu_{e}$ coincides with $\sigma$. Nevertheless there are semigroups $T(X)$ with the property that $\mu_{e} \neq \sigma$. We discuss such a semigroup in what follows.

EXAMPLE (2.21). Let $R$ denote the space of real numbers with the usual topology, let $R^{-}$denote the subspace of non-positive reals and let $R^{+}$denote the subspace of non-negative reals. Let $T(R)$ consist of all continuous functions which map $R^{-}$ 
homeomorphically onto $R^{+}$and also $R^{+}$homeomorphically onto $R^{+}$together with all the constant functions and the identity map. We show first that $\mu_{e}=\Delta(R)$. Note that $(0, x) \notin \mu$ for $x \neq 0$ since for any $\{a, b\}$ such that $a \neq 0 \neq b$, no $f \in T(R)$ can map $\{a, b\}$ onto $\{0, x\}$. Specifically, no map in $T(R)$ other than $\langle 0\rangle$ sends a nonzero element into zero. On the other hand, if $x \neq y$ and $x \neq 0 \neq y$, then $(x, y) \notin \mu$ since we can take a pair $\{0, a\}$ with $a \neq 0$ and there is no $f \in T(R)$ such that $f$ maps $\{0, a\}$ onto $\{x, y\}$. This is because $f(0)=0$ for each nonconstant function $f \in T(R)$. Thus, $\mu=\Delta(R)$ which means $\mu_{e}=\Delta(R)$.

However, $\sigma \neq \Delta(R)$. Let $a, b>0$. We will show that $(a, b) \in \eta_{e}(x, y)$ for all distinct $x, y \in R$. This follows easily if $x \neq 0 \neq y$ for in that case, one shows in a routine manner that $(a, b) \in \eta_{e}(x, y)$. Consider the case where one of the points, say $x$, is equal to 0 . Then we must show that $(a, b) \in \eta_{e}(0, y)$. Evidently, there exists an $f \in T(R)$ such that $f(y)=a$ and, of course, we must have $f(0)=0$. Similarly, there exists a $g \in T(R)$ such that $g(y)=b$ and $g(0)=0$. Thus, $(0, a),(0, b) \in \eta(0, y)$ and hence $(a, b) \in \eta_{e}(0, y)$. This proves that $\sigma \neq \Delta(R)$ and, in fact, it is easy to see that

$$
\sigma=\{(a, b) \in R \times R: a>0 \text { and } b>0\} \cup \Delta(R) .
$$

Thus, $X$ does have a smallest proper $T$-equivalence $\sigma$ but it is not $\mu_{e}$.

3. The smallest proper congruence on $S(X)$. In this section (actually throughout the remainder of the paper), $X$ will be a topological space and the semigroup under consideration will be $S(X)$ the semigroup of all continuous selfmaps of $X$. In order to be consistent with our notation we will, from this point on, use the term $S$-equivalence in place of the term $T$-equivalence which we used throughout the previous section and the complete lattice of $S$-equivalences on $X$ will be denoted by $\operatorname{Seq}(X)$. We will see that it is really quite rare for $S(X)$ not to have a smallest proper congruence. We will describe this congruence in various instances by first determining the smallest proper $S$-equivalence $\sigma$ on $X$, which, in all the cases we consider here, coincides with $\mu_{e}$ and then appealing to Corollary (2.15), which tells us that the smallest proper congruence on $S(X)$ is $C(\sigma)=\{(\langle x\rangle,\langle y\rangle) \in X \times X:(x, y) \in \sigma\} \cup \Delta(S(X))$. We begin our considerations by defining a relation $\mathscr{G}$ on $X$ which differs from $\Delta(X)$ for spaces which are not $T_{0}$. Let

$\mathscr{G}=\{(x, y) \in X \times X: x$ and $y$ belong to precisely the same open subsets of $X\}$.

$\mathscr{G}$ is evidently an equivalence relation on $X$ and regarding it we have the following.

Theоrem (3.1). Suppose $X$ is not $T_{0}$. Then $\mathscr{G}$ is the smallest proper $S$-equivalence on $X$. Consequently, $C(\mathscr{G})$ is the smallest proper congruence on $S(X)$, where

$$
C(\mathscr{G})=\{(\langle x\rangle,\langle y\rangle):(x, y) \in \mathscr{G}\} \cup \Delta(S(X)) .
$$

Proof. We show that $\mathscr{G}=\mu_{e}$. Suppose $(x, y) \in \mathscr{G}$ and let $a$ and $b$ be distinct points of $X$. Define $f(a)=x$ and $f(z)=y$ for $z \neq a$. Let $G$ be any open subset of $X$. Since $x$ and $y$ belong to precisely the same open sets, either $x, y \in G$ or $x, y \notin G$. In the former case, $f^{-1}[G]=X$ while in the latter, $f^{-1}[G]=\varnothing$. Thus, $f$ is continuous and $(x, y) \in \mu \subseteq \mu_{e}$, 
which implies $\mathscr{G}_{\subseteq} \mu_{e}$. Since $X$ is not $T_{0}$, it contains two distinct points $a$ and $b$ which belong to precisely the same open sets so that $\mathscr{G} \neq \Delta(X)$. Let $(x, y) \in \mu$. Then some $f \in S(X)$ maps $\{a, b\}$ onto $\{x, y\}$. It follows that $x$ and $y$ must belong to the same open sets also and thus $(x, y) \in \mathscr{G}$. Since $\mathscr{G}$ is an equivalence relation, it follows that $\mu_{e} \subseteq \mathscr{G}$. We have now shown that $\mu_{e}=\mathscr{G} \neq \Delta(X)$ so it follows from Theorem (2.19) that $\sigma=\mu_{e}=\mathscr{G}$ and the remaining assertions now follow from Corollary (2.15).

Definition (3.2). A topological space is said to be Aleksandrov discrete if it is $T_{0}$ and arbitrary intersections of open sets are open.

P. S. Aleksandrov has reserved the term discrete for what we here call Aleksandrov discrete spaces [1, p. 28]. Such spaces arise naturally in the study of partially ordered sets and isotone maps. See also [6]. When we use the term discrete, however, we will mean it in the sense that has come to be more commonly accepted. That is, all subsets are open.

For an Aleksandrov discrete space $X$ and a point $x \in X$, we denote by $G_{x}$ the intersection of all open sets containing $x$. Define a relation $\mathscr{A}$ on $X$ by

$$
\mathscr{A}=\left\{(x, y) \in X \times X: G_{x} \cap G_{y} \neq \varnothing\right\}
$$

and let $\mathscr{A}_{e}$ be the smallest equivalence relation on $X$ containing $\mathscr{A}$.

Theorem (3.3). Let $X$ be an Aleksandrov discrete space. Then $X$ has a smallest proper $S$-equivalence $\sigma$. If $X$ is discrete then $\sigma=X \times X$ and if $X$ is not discrete then $\sigma=\mathscr{A}_{e}$. Consequently, $S(X)$ has a smallest proper congruence $C(\sigma)$, where

$$
C(\sigma)=\{(\langle x\rangle,\langle y\rangle):(x, y) \in X \times X\} \cup \Delta(S(X))
$$

if $X$ is discrete and

$$
C(\sigma)=\left\{(\langle x\rangle,\langle y\rangle):(x, y) \in \mathscr{A}_{e}\right\} \cup \Delta(S(X))
$$

if $X$ is not discrete.

Proof. If $X$ is discrete, there are only two $S$-equivalences on $X, \Delta(X)$ and $X \times X$, so it is evident that $\sigma=X \times X$ in this case. We consider the case where $X$ is not discrete and we show that $\mu_{e}=\mathscr{A}_{e}$ which, of course, means $\sigma=\mathscr{A}_{e}$. Let $(x, y) \in \mu$. Since $X$ is not discrete, some $G_{a}$ contains a point $b \neq a$ and therefore, $G_{b} \subseteq G_{a}$. Let $f$ be any function in $S(X)$ which maps $\{a, b\}$ onto $\{x, y\}$. Then

$$
G_{b} \subseteq f^{-1}\left[G_{x}\right] \cap f^{-1}\left[G_{y}\right]=f^{-1}\left[G_{x} \cap G_{y}\right],
$$

which means $G_{x} \cap G_{y} \neq \varnothing$. Thus $(x, y) \in \mathscr{A}$ and it follows that $\mu_{e} \subseteq \mathscr{A}_{e}$.

Now suppose that $(x, y) \in \mathscr{A}$ and let $a$ and $b$ be any two distinct points of $X$. Then there is a $z \in G_{x} \cap G_{y}$ and hence $G_{z} \subseteq G_{x} \cap G_{y}$. Moreover, $G_{a} \neq G_{b}$ since $X$ is $T_{0}$. It may be that $G_{a} \ddagger G_{b}$ and $G_{b} \notin G_{a}$ or that one of the sets is contained in the other. If the latter is the case, we may assume that $G_{a} \subseteq G_{b}$. Now define

and

$$
f(w)=z \text { if } G_{w} \subseteq G_{a}
$$

$$
f(w)=x \text { if } \quad G_{w} \nsubseteq G_{a} .
$$


Sets of the form $G_{v}, v \in X$ form a basis so it is sufficient to consider these in checking continuity. If $x \in G_{v}$ then $z, x \in G_{x} \subseteq G_{v}$ and $f^{-1}\left[G_{v}\right]=X$. It remains to consider the case where $z \in G_{v}$ and $x \notin G_{v}$. In this case,

$$
f^{-1}\left[G_{v}\right]=\bigcup\left\{G_{w}: G_{w} \subseteq G_{a}\right\}
$$

so that $f$ is continuous. Furthermore, $f$ maps $\{a, b\}$ onto $\{z, x\}$, which means $(z, x) \in \mu$. Similarly, $(z, y) \in \mu$ and thus $(x, y) \in \mu_{e}$. This implies that $\mathscr{A}_{e} \subseteq \mu_{e}$ and hence $\sigma=\mu_{e}=\mathscr{A}_{e}$ when $X$ is a nondiscrete Aleksandrov discrete space. Again, the remaining assertions follow from Corollary (2.15).

COROLlaRY (3.4). The semigroup of every finite space has a smallest proper congruence.

Proof. Let $X$ be a finite space. If it is not $T_{0}$, the conclusion follows from Theorem (3.1). If it is $T_{0}$, it is an Aleksandrov discrete space and the conclusion follows from Theorem (3.3).

Remark. Recall that the Sierpinski space is the two element space $\{a, b\}$ where the open sets are $\varnothing,\{a\}$ and $\{a, b\}$. Let $X$ be any infinite discrete space and let $Y$ be the discrete union of $X$ with the Sierpinski space. Both $X$ and $Y$ are Aleksandrov discrete spaces and topologically, they appear to be quite similar. Specifically, $Y$ is just about as close to being discrete as it could be. Nevertheless the smallest proper $S$-equivalences in the two cases differ radically. According to Theorem (3.3), the smallest proper $S$-equivalence on $X$ is $X \times X$ while the smallest proper $S$-equivalence on $Y$ is $\{(a, b)$, $(b, a)\} \cup \Delta(Y)$.

The next equivalence relation we introduce will be quite familiar. For any topological space $X$, let

$$
\mathscr{P}=\{(x, y) \in X \times X ; \text { there is a path in } X \text { from } x \text { to } y\} .
$$

We recall that a space $X$ is said to be totally separated if for distinct points $x, y \in X$, there exists a clopen set $H$ with $x \in H$ and $y \notin H$.

THEOREM (3.5). Suppose $X$ is either totally separated or is completely regular, Hausdorff and contains an arc. Then $X$ has a smallest proper $S$-equivalence $\sigma$. In the former case, $\sigma=X \times X$ while in the latter case, $\sigma=\mathscr{P}$. Consequently. $S(X)$ has a smallest proper congruence $C(\sigma)$, where

$$
C(\sigma)=\{(\langle x\rangle,\langle y\rangle):(x, y) \in X \times X\}
$$

when $X$ is totally separated and

$$
C(\sigma)=\{(\langle x\rangle,\langle y\rangle):(x, y) \in \mathscr{P}\} \cup \Delta(S(X))
$$

when $X$ is completely regular, Hausdorff and contains an arc.

Proof. It follows easily that if $X$ is totally separated, then $\mu_{e}=\mu=X \times X$.

Suppose $X$ is completely regular, Hausdorff and contains an arc. We show that in this 
case $\mu_{e}=\mathscr{P}$. Let $(x, y) \in \mu$ and let $a$ and $b$ be endpoints of an $\operatorname{arc} A$ in $X$. Then there exists an $f \in S(X)$ such that $f(a)=x$ and $f(b)=y$. Thus, $(x, y) \in \mathscr{P}$, and it follows that $\mu_{e} \subseteq \mathscr{P}$.

On the other hand, suppose $(x, y) \in \mathscr{P}$. Then there exists a continuous function $f$ from the closed unit interval $I=[0,1]$ such that $f(0)=x$ and $f(1)=y$. For any two distinct points $a, b \in X$, there exists a continuous map $g$ from $X$ into $I$ such that $g(a)=0$ and $g(b)=1$. Thus, $f \circ g \in S(X), f \circ g(a)=x$ and $f \circ g(b)=y$ which means $(x, y) \in \mu_{e}$. Thus $\mu_{e}=\mathscr{P}$ and the remaining assertions follow as in the previous proofs.

EXAmple (3.6). The results of this section tell us that for a great many spaces $X, S(X)$ does have a smallest proper congruence. Nevertheless, there are completely regular Hausdorff spaces $X$ for which $S(X)$ does not have a smallest proper congruence. In [2], J. de Groot proved the existence of $2^{c} 1$-dimensional, connected, locally connected subspaces of the Euclidean plane with the property that the only continuous maps from one such space into another are the constant maps and for any such space $X$, the only continuous selfmaps are the constant maps and the identity map. $S(X)$ is therefore a left zero semigroup with identity containing $c$ elements. It follows easily from Theorem (2.18) that $X$ has no smallest proper $S$-equivalence and thus $S(X)$ has no smallest proper congruence. However, $S \operatorname{eq}(X)$ does contain many atoms. In fact, if $x, y \in X$ and $x \neq y$, then $\eta_{e}(x, y)$ will be an atom since (2.16.2) is vacuously satisfied and

$$
\eta_{e}(x, y)=\{(x, y),(y, x)\} \cup \Delta(X) .
$$

The atoms of Cong $(S(X))$ are therefore all the congruences of the form

$$
\{(\langle x\rangle,\langle y\rangle),(\langle y\rangle,\langle x\rangle)\} \cup \Delta(S(X))
$$

where $x$ and $y$ are distinct. Of course, $X$ is completely regular and Hausdorff but it contains no arcs.

For each of de Groot's spaces $X, S(X)$ contains as few continuous functions as possible. One can use these spaces to form another which has a greater variety of continuous selfmaps but nevertheless, the semigroup has no smallest proper congruence. Let $\left\{Y_{\alpha}\right\}_{\alpha \in \Lambda}$ be the collection of de Groot's spaces and let $X$ be the discrete union of all those spaces. As we noted previously, the cardinality of $\Lambda$ is $2^{c}$. Let $\mathscr{T}_{\Lambda}$ denote the semigroup of all selfmaps of $\Lambda$. The semigroup $S(X)$ contains a copy of $\mathscr{T}_{\Lambda}$. To see this, choose $y_{\alpha} \in Y_{\alpha}$ for each $\alpha \in \Lambda$ and for $f \in \mathscr{T}_{\Lambda}$ and $x \in Y_{\alpha}$, define $\varphi(f)(x)=y_{f(\alpha)}$. One easily checks that $\varphi$ is an isomorphism from $\mathscr{T}_{\Lambda}$ into $S(X)$. To see that $X$ has no smallest proper $S$-equivalence, choose two distinct points $x$ and $y$ both in the same $Y_{\alpha}$. Let $f$ be any function in $S(X)$ and let $f_{\alpha}$ be the restriction of $f$ to $Y_{\alpha}$. If $f_{\alpha}$ maps $Y_{\alpha}$ into some $Y_{\beta}, \beta \neq \alpha$, then $f_{\alpha}$ is a constant map. If $f_{\alpha}$ maps $Y_{\alpha}$ into $Y_{\alpha}$, it is either a constant map or the identity map. This means that either $(f(x), f(y))=(x, y)$ or $(f(x), f(y))=(z, z)$ for some $z \in X$ and hence that

It readily follows that

$$
\eta_{e}(x, y)=\{(x, y),(y, x)\} \cup \Delta(X) \text {. }
$$

$$
\sigma=\bigcap\left\{\eta_{e}(x, y):(x, y) \in X \times X, x \neq y\right\}=\Delta(X)
$$


and therefore $X$ has no smallest proper $S$-equivalence by Theorem (2.18). Not every $S$-equivalence of the form $\eta_{e}(x, y)$ is an atom for this particular space but it readily follows from Theorem (2.16) that for this particular space $X$, the atoms of $S$ eq $(X)$ are precisely those $\eta_{e}(x, y)$ where $x$ and $y$ are distinct and both belong to the same component. It is perhaps worthwhile to emphasize the fact that if we want to show that a semigroup has a smallest proper congruence and we are able to show that $\mu_{e} \neq \Delta(X)$, then that is sufficient. But it is not enough to show that $\mu_{e}=\Delta(X)$ in order to conclude that $S(X)$ does not have a smallest proper congruence. However, as Theorem (2.18) tells us, we can draw that conclusion any time we are able to show that $\sigma=\Delta(X)$.

4. Congruences and connectedness. $K(X)$ denotes the kernel of $S(X)$ and $\pi(K(X))$ is the Rees factor congruence which identifies all elements in $K(X)$ to a point. It is easily verified that for any space $X$ whatsoever, $K(X)$ is the collection of constant functions. Whether or not $\pi(K(X))$ is the smallest proper congruence on $S(X)$ depends, in many instances, upon the connectedness of $X$. Our first result is an easy consequence of Theorem (3.5).

THEOREM (4.1). Suppose $X$ is completely regular, Hausdorff and contains an arc. Then $\sigma(K(X))$ is the smallest proper congruence on $S(X)$ if and only if $X$ is pathwise connected.

Proof. According to Theorem (3.5),

whereas

$$
C(\sigma)=\{(\langle x\rangle,\langle y\rangle:(x, y) \in \mathscr{P}\} \cup \Delta(S(X))
$$

$$
\pi(K(X))=\{(\langle x\rangle,\langle y\rangle:(x, y) \in X \times X\} \cup \Delta(S(X)) .
$$

Evidently, $\pi(K(X))=C(\sigma)$ if and only if $X$ is pathwise connected.

Definition (4.2). A space $X$ has the internal extension property if every continuous function from a closed subset of $X$ into $X$ can be extended to a continuous selfmap of $X$.

Any absolute retract has this property as well as any 0-dimensional metric space [3, p. 281]. In particular, Euclidean $N$-cells, Euclidean $N$-spaces, Hilbert space and the Hilbert cube all have it. The space

$$
X=\left\{(x, y) \in R^{2}: 1 \leq x^{2}+y^{2} \leq 5\right\}
$$

does not have it. Let $h$ be any homeomorphism from

$$
B_{1}=\left\{(x, y) \in R^{2}:(x-3)^{2}+y^{2}=1\right\}
$$

onto

$$
B_{2}=\left\{(x, y) \in R^{2}: x^{2}+y^{2}=5\right\}
$$

and suppose $h$ can be extended to a continuous selfmap $h_{1}$ of $X$. Let

$$
D=\left\{(x, y) \in R^{2}:(x-3)^{2}+y^{2} \leq 1\right\}
$$

and let $h_{2}=h_{1} \mid D$. Now let $k$ be any continuous selfmap of $X$ which retracts $X$ onto $B_{2}$. 
Then $h^{-1} \circ k \circ h_{2}$ retracts $D$ onto $B_{1}$ contradicting a well known theorem of Brouwer, and therefore, we see that $h$ cannot be extended to a continuous selfmap of $X$.

In our next result, we encounter the notion of a prime ideal. We will assume here that a prime ideal of a semigroup is a proper subset of that semigroup.

THEOREM (4.3). Let $X$ be any locally connected, compact, $N$-dimensional subspace of Euclidean $N$-space whose components all have the internal extension property. Then the following statements are equivalent.

$\pi(K(X))$ is the smallest proper congruence on $S(X)$.

$S(X)$ has a largest proper congruence.

$S(X)$ has no prime ideals.

$X$ is pathwise connected.

Proof. It is immediate from Theorem (4.1) that (4.3.1) and (4.3.4) are equivalent. If $X$ is pathwise connected, then $S(X)$ has a largest proper congruence in view of Corollary 6 of [4] so that (4.3.4) implies (4.3.2). We show next that (4.3.2) implies (4.3.4). Suppose, to the contrary, that $X$ is not pathwise connected. Each component of $X$ is pathwise connected since each is a Peano continuum so that the path components and the components of $X$ coincide. Let

$$
J=\{f \in S(X): \operatorname{Ran} f \cap A=\varnothing \text { for at least one component } A \text { of } X\},
$$

where $\operatorname{Ran} f$ denotes the range of $f$. Since $X$ is locally connected, its components are open so it can have only finitely many since it is also compact. One verifies in a straightforward manner that $J$ is a prime ideal of $S(X)$. Consequently,

$$
M=(J \times J) \cup((S(X)-J) \times(S(X)-J))
$$

is a congruence on $S(X)$. Now, $M$ is evidently a maximal proper congruence so if $S(X)$ is to have a largest proper congruence, it must be $M$. Declare $(f, g) \in Q$ if whenever $Y$ is a subspace homeomorphic to $X$ and one of the two functions is injective on $Y$, then the two functions agree on $Y$. One verifies that $Q$ is a proper congruence on $S(X)$. Let $\left\{A_{j}\right\}_{j=1}^{N}$ denote the components of $X$, choose $a_{j} \in A_{j}$ for $1 \leq j \leq N$ and define $f(x)=a_{j}$ for $x \in A_{j}$. Next, define a function $g$ by $g(x)=a_{j}$ for $x \in A_{j}, j \neq 1$ and $g(x)=a_{2}$ whenever $x \in A_{1}$. Then $(f, g) \in Q-M$ and we have a contradiction. Thus, we have shown that (4.3.2) implies (4.3.4) so the two are equivalent.

We have seen that if $X$ is not pathwise connected, then $S(X)$ contains a prime ideal (the ideal $J$ defined previously) so that (4.3.3) implies (4.3.4). We show that (4.3.4) implies (4.3.3). Suppose, to the contrary, that $S(X)$ contains a prime ideal $P$. Then

$$
V=(P \times P) \cup((S(X)-P) \times(S(X)-P))
$$

is a proper congruence on $S(X)$. Next, for any $f, g \in S(X)$, let $(f, g) \in W$ if and only if whenever either of the functions is injective on any subspace of $X$ which is homeomorphic to the Euclidean $N$-cell, $I^{N}$, then the two functions agree on that subspace. Corollary 6 of 
[4] assures us that $W$ is the largest proper congruence on $S(X)$. But $V \nsubseteq W$. To see this, let $h$ and $k$ be any two homeomorphisms from $X$ onto $X$ which differ on some subspace of $X$ which is homeomorphic to $I^{N}$. Then $h, k \in S(X)-P$ so that $(h, k) \in V-W$. This contradiction leads us to to conclude that when $X$ is pathwise connected, then $S(X)$ can have no prime ideals. That is, (4.3.4) implies (4.3.3) and the proof is complete.

\section{REFERENCES}

1. P. S. Aleksandrov, Combinatorial topology (Graylock Press, 1956). $80-102$.

2. J. de Groot, Groups represented by homeomorphism groups, I, Math. Ann. 138 (1959),

3. K. Kuratowski, Topology, Vol. I (Academic Press, 1966).

4. K. D. Magill, Jr., The largest proper congruence on $S(X)$, Internat. J. Math. Math. Sci. (7) 4 (1984), 663-666.

5. K. D. Magill, Jr., On a family of ideals of $S(X)$, Semigroup Forum 34 (1987), 321-339.

6. M. C. Thornton, Semigroups of isotone selfmaps on partially ordered sets, J. London Math. Soc. (2) 14 (1976), 545-553.

Fachbereich Mathematik

Technische Hochschule Darmstadt

SCHLOSSGARTENSTR. 7

D-6100 Darmstadt, Germany (FRG)

SUNY AT BUFFaLO

106 Diefendorf Hall

Buffalo, New York

14214-3093

U.S.A. 